\title{
NEARLY REPRESENTABLE OPERATORS
}

\author{
R. KAUFMAN, MINOS PETRAKIS, LAWRENCE H. RIDDLE AND J. J. UHL, JR.
}

\begin{abstract}
Among Bourgain's many remarkable theorems is one from 1980 which states that if $T$ is a non-Dunford-Pettis operator from $L_{1}[0,1]$ into an arbitrary Banach space, then there is a Dunford-Pettis operator $D$ from $L_{1}[0,1]$ into $L_{1}[0,1]$ such that the composition $T \circ D$ is not Bochner representable. This theorem sets up the following question: What are the operators $T$ from $L_{1}[0,1]$ into a Banach space $X$ such that $T \circ D$ is Bochner representable for all Dunford-Pettis operators $D: L_{1}[0,1] \rightarrow L_{1}[0,1]$ ? We call such an operator nearly representable. In view of Bourgain's theorem, all nearly representable operators are Dunford-Pettis. If $X$ is a Banach space such that all nearly representable operators are, in addition, Bochner representable, then we say $X$ has the near Radon-Nikodym property (NRNP) and ask which Banach spaces have the NRNP? This paper is an attempt to provide at least partial answers to these questions.

The first section collects terminology, gives the introductory results and shows that the NRNP is a three space property. The second section studies a continuity property that implies near representability. Finally, the third section contains the main result of the paper, Theorem 15 , which states that if $T: L_{1}[0,1] \rightarrow L_{1}[0,1]$ is a nonrepresentable operator, there exists a DunfordPettis operator $D: L_{1}[0,1] \rightarrow L_{1}[0,1]$ such that $T \circ D$ is also nonrepresentable. This implies that the NRNP is shared by $L_{1}[0,1]$, lattices not containing $c_{0}$, and $L_{1}(\mathrm{~T}) / H_{0}^{1}$.
\end{abstract}

\section{Preliminaries}

One operator that has an intriguing history is $V: L_{1}[0,1] \rightarrow C[0,1]$, the Volterra operator, defined for $f \in L_{1}[0,1]$ by

$$
V f(t)=\int_{0}^{t} f d \mu, \quad 0 \leq t \leq 1 .
$$

Probably the most intriguing fact about this operator is that it is completely continuous, i.e., it sends weakly compact (uniformly integrable) sets in $L_{1}[0,1]$ into compact (equicontinuous) sets in $C[0,1]$. Once this fact is realized, it is

Received by the editors November 5, 1987.

1980 Mathematics Subject Classification (1985 Revision). Primary 47A65, 47B25, 28 A33.

Key words and phrases. Dunford-Pettis operators, Bochner representable operators, singular measures, Radon-Nikodym property, semi-embeddings.

Some of the theorems of this paper are from the doctoral dissertation of the second author.

The work of the first author was supported in part by an NSF grant.

The work of the third author was supported in part by Emory University Research Committee Grant 2-50113 and a Research Committee Summer Fellowship. 
very easy for one to see

Property (a). The Volterra operator $V$ is a Dunford-Pettis operator that is not Bochner representable (Lewis [11]).

Consequently

Property (b). When restricted to $C[0,1]$, the resulting operator is a positive compact absolutely summing operator that is not nuclear (Diestel-Uhl [5, VI.4.4]).

Less well-known but just as interesting is an additional property of $V$ : $L_{1}[0,1] \rightarrow C[0,1]$ discovered by Bourgain [1].

Property (c). If $D: L_{1}[0,1] \rightarrow L_{1}[0,1]$ is any Dunford-Pettis operator, then the composition $V \circ D: L_{1}[0,1] \rightarrow C[0,1]$ is Bochner representable.

This paper is devoted to the study of operators from $L_{1}[0,1]$ to a Banach space that share this last property.

Now let us pause to fix some notation and terminology. Throughout this paper $X$ and $Y$ are Banach spaces and $\mu$ is Lebesgue measure on $[0,1]$. A bounded linear operator $T: L_{1}[0,1] \rightarrow X$ is Bochner representable if there is a bounded measurable function $g:[0,1] \rightarrow X$ such that

$$
T f=\int_{[0,1]} f g d \mu
$$

for all $f \in L_{1}[0,1]$. A bounded linear operator $D: L_{1}[0,1] \rightarrow X$ is a DunfordPettis operator if $D$ sends weakly compact sets into norm compact sets. It is easy to see (Diestel-Uhl [5, III.2.11]) that all Bochner representable operators on $L_{1}[0,1]$ are Dunford-Pettis operators, but the converse is not true (thanks to the existence of the Volterra operator $V$ defined in the opening paragraphs).

A Banach space $X$ has the Radon-Nikodym property if all bounded linear operators from $L_{1}[0,1]$ to $X$ are Bochner representable. Bourgain [1] has shown that a Banach space has the Radon-Nikodym property if and only if all Dunford-Pettis operators from $L_{1}[0,1]$ to $X$ are Bochner representable. The following definitions isolate the topics of this paper.

Definition 1. A bounded linear operator $T: L_{1}[0,1] \rightarrow X$ is nearly representable if for each Dunford-Pettis operator $D: L_{1}[0,1] \rightarrow L_{1}[0,1]$ the composition $T \circ D: L_{1}[0,1] \rightarrow X$ is Bochner representable.

By Property (c) above, the Volterra operator $V$ is nearly representable.

Definition 2. A Banach space $X$ has the near Radon-Nikodym property (NRNP) if every nearly representable operator from $L_{1}[0,1]$ to $X$ is Bochner representable.

Thanks to the Volterra operator $V$, we see that $C[0,1]$ fails the NRNP. The main result of this paper, Theorem 15 in $\S 3$, is that $L_{1}[0,1]$ has the NRNP. Before showing that, however, we give some general results on nearly representable operators and the NRNP.

A few facts become immediate. 
Fact 3. Any representable operator from $L_{1}[0,1]$ to $X$ is nearly representable. Proof. This is not an immediate consequence of the definitions, but it is an immediate consequence of the Lewis-Stegall theorem (see Diestel-Uhl [5, III.1.8]). This theorem says that an operator $T: L_{1}[0,1] \rightarrow X$ is Bochner representable if and only if it factors through $l_{1}$. Thus if $T: L_{1}[0,1] \rightarrow X$ is Bochner representable, then it factors through $l_{1}$ and hence so does the composition $T \circ D$ for any bounded linear operator $D: L_{1}[0,1] \rightarrow L_{1}[0,1]$, and in particular for any Dunford-Pettis operator $D: L_{1}[0,1] \rightarrow L_{1}[0,1]$. Therefore $T \circ D$ is Bochner representable, which proves Fact 3.

An immediate consequence is

Fact 4. If a Banach space has the RNP, then it has the NRNP.

In his paper [1], Bourgain proved that if a bounded linear operator $T$ : $L_{1}[0,1] \rightarrow X$ is not Dunford-Pettis, then there is a Dunford-Pettis operator $D: L_{1}[0,1] \rightarrow L_{1}[0,1]$ such that $T \circ D$ is not Bochner representable. Or equivalently,

Fact 5. The nearly representable operators on $L_{1}[0,1]$ are all Dunford-Pettis operators.

Nearly representable operators have a geometric characterization in terms of bounded trees. It is well known that an operator $T: L_{1}[0,1] \rightarrow X$ can be specified through its associated martingale

$$
g_{n}=\sum_{E \in \Pi_{n}} \frac{T\left(\chi_{E}\right)}{\mu(E)} \chi_{E}
$$

where $\Pi_{n}$ is the $n$th dyadic partition of $[0,1]$. On the other hand, the martingale $\left(g_{n}\right)$ is specified by the tree

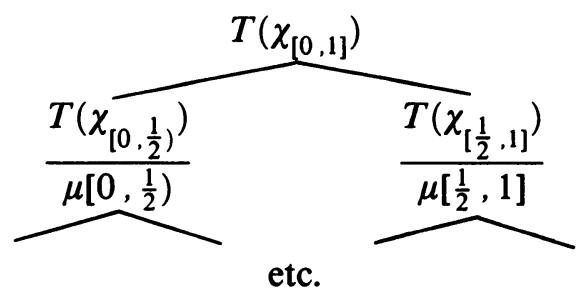

An operator $T: L_{1}[0,1] \rightarrow X$ is Dunford-Pettis if and only if its tree or martingale is Pettis Cauchy; i.e.

$$
\lim _{n, m} \sup _{\left\|x^{*}\right\| \leq 1} \int_{[0,1]}\left|x^{*} g_{n}-x^{*} g_{m}\right| d \mu=0 .
$$

(For a proof, see Riddle-Uhl [18].) The same operator is Bochner representable if and only if its tree or martingale is Bochner Cauchy; i.e.

$$
\lim _{n, m} \int_{[0,1]}\left\|g_{n}-g_{m}\right\|_{X} d \mu=0 .
$$

Thus the following fact is now evident. 
Fact 6. An operator $T: L_{1}[0,1] \rightarrow X$ is nearly representable if and only if it maps bounded Pettis Cauchy trees into Bochner Cauchy trees.

(This fact allows one to define the notion of near representability for any operator $T: X \rightarrow Y$ in terms of its action on bounded Pettis Cauchy trees; for more on this see Petrakis [16].)

The last result of this section shows that the near Radon-Nikodym property is a genuine "three space property." The proof makes use of a clever factoring and lifting argument suggested to us indirectly by Edgar via Dowling [7].

Theorem 7. Let $X$ be a Banach space and $Y$ be a closed subspace of $X$. If $Y$ and $X / Y$ have the near Radon-Nikodym property, then $X$ has the near RadonNikodym property.

Proof. Let $T: L_{1}[0,1] \rightarrow X$ be nearly representable and let $q: X \rightarrow X / Y$ be the quotient map. Clearly $q \circ T$ is nearly representable and since $X / Y$ has the near Radon-Nikodym property, we see that $q \circ T$ is Bochner representable; consequently by the Lewis-Stegall theorem the operator $q \circ T$ factors through $l_{1}$. Choose bounded linear operators $A: L_{1}[0, i] \rightarrow l_{1}$ and $B: l_{1} \rightarrow X / Y$ such that $q \circ T=B \circ A$. Because $q$ is onto, the "lifting property" for $l_{1}$ (see Lindenstrauss-Tzafriri [12, p. 107]) produces a bounded linear operator $\widetilde{B}: l_{1} \rightarrow X$ such that $B=q \circ \widetilde{B}$.

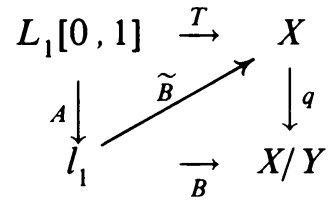

Let $S=T-\widetilde{B} A$ and note that $q S=q T-q \widetilde{B} A=q T-B A=0$. Therefore $S$ maps all of $L_{1}[0,1]$ into $Y$.

Take a Dunford-Pettis operator $D: L_{1}[0,1] \rightarrow L_{1}[0,1]$ and compute $S D=$ $T D-\widetilde{B} A D$. Now $T D, \widetilde{B} A$, and $\widetilde{B} A D$ factor through $l_{1}$ and hence all three are Bochner representable. Therefore $S \circ D$ is Bochner representable and hence $S$ is nearly representable. Since $S\left(L_{1}[0,1]\right) \subseteq Y$ and $Y$ has the near RadonNikodym property, we find that $S$ is itself Bochner representable. This proves that $T=S+\widetilde{B} A$ is Bochner representable because both $S$ and $\widetilde{B} A$ are Bochner representable, and completes the proof.

\section{2. $M_{0}$-CONTINUITY}

This section attempts to capitalize on the properties of the Volterra operator $V: L_{1}[0,1] \rightarrow C[0,1]$ defined for $f \in L_{1}[0,1]$ by

$$
V f(t)=\int_{0}^{t} f d \mu, \quad 0 \leq t \leq 1 .
$$

In [1], Bourgain proved that this operator is nearly representable and this fact can be used to recognize a variety of nearly representable operators. To this 
end, define the norm \|\|$\cdot\|\|_{0}$ for $f \in L_{1}[0,1]$ by

$$
\left\||| f\left|\|_{0}=\sup _{I}\right| \int_{I} f d \mu \mid\right.
$$

where the supremum is taken over all subintervals $I$ of $[0,1]$. Call this norm the $M_{0}$-norm of $f$ and note that although it agrees with the usual $L_{1}[0,1]$ norm for positive functions, it is not equivalent to the $L_{1}[0,1]$-norm. (In fact, on weakly compact sets it agrees with the weak topology of $L_{1}[0,1]$ !)

Definition 8. A bounded linear operator $T: L_{1}[0,1] \rightarrow X$ is $M_{0}$-continuous if it is continuous for the $M_{0}$-norm on $L_{1}[0,1]$.

The main result of this section is

Theorem 9. If $X$ is an arbitrary Banach space, then any bounded linear operator $T: L_{1}[0,1] \rightarrow X$ that is $M_{0}$ continuous is nearly representable.

Proof. Let $T: L_{1}[0,1] \rightarrow X$ be a bounded linear operator that is $M_{0}$-continuous. Let $Y$ be the subspace of $C[0,1]$ consisting of all functions $\phi_{f}$ of the form

$$
\phi_{f}(t)=\int_{0}^{t} f d \mu, \quad 0 \leq t \leq 1,
$$

for some $f \in L_{1}[0,1]$. Define $\tilde{L}: Y \rightarrow X$ by $\widetilde{L}\left(\phi_{f}\right)=T f$ and note that

$$
\|\tilde{L}\|=\sup _{\left\|\phi_{f}\right\|_{\infty} \leq 1}\left\|\tilde{L}\left(\phi_{f}\right)\right\|=\sup _{\left\|\phi_{f}\right\|_{\infty} \leq 1}\|T f\| \leq 2 \sup _{\|f\|_{0} \leq 1}\|T f\|<\infty
$$

since $\|f\|\left\|_{0} \leq 2\right\| \phi_{f} \|_{\infty}$ and $T$ is $M_{0}$-continuous.

This shows that $L$ is bounded on $Y$ and therefore has a bounded extension to the closure $\bar{Y}$ of $Y$ in $C[0,1]$. A moment's reflection shows that $\bar{Y}$ is of codimension 1 in $C[0,1]$ and hence there is a bounded linear operator $L: C[0,1] \rightarrow X$ such that $L\left(\phi_{f}\right)=\widetilde{L}\left(\phi_{f}\right)=T f$ for all $f \in L_{1}[0,1]$.

Putting this together shows that $T$ admits the factorization $T=L V$

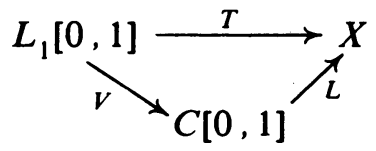

where $V$ is the Volterra operator. By Property (c), the operator $V$ is nearly representable. Thus if $D: L_{1}[0,1] \rightarrow L_{1}[0,1]$ is Dunford-Pettis, then $V \circ D$ is Bochner representable and hence $T \circ D=L \circ V \circ D$ is Bochner representable. This shows $T$ is nearly representable and this completes the proof.

This theorem sets up some interesting examples and corollaries.

Example 10. Let $\left(I_{n}\right)$ be any sequence of subintervals of $[0,1]$ such that $\lim _{n} \mu\left(I_{n}\right)=0$. The operator $T: L_{1}[0,1] \rightarrow c_{0}$ defined by $T f=\left(\int_{I_{n}} f d \mu\right)$ is nearly representable. If $\left(\chi_{I_{n}}\right)$ does not converge to zero almost everywhere, then this operator is not representable. 
Proof. Evidently $T$ is $M_{0}$-continuous and therefore nearly representable. On the other hand, such an operator is Bochner representable if and only if $\lim _{n} \chi_{I_{n}}$ $=0$ almost everywhere (Diestel-Uhl [5, p. 75]).

For a more specific example, we see that Pettis integration with respect to Hagler's Murphy's Pub function (see Diestel-Uhl [5, II.1.7]) defines an operator from $L_{1}[0,1]$ to $c_{0}$ that is nearly representable but not representable.

Example 10 combined with the next example shows that the nearly representable operators from $L_{1}[0,1]$ to $c_{0}$ sit strictly between the representable operators and the Dunford-Pettis operators. The next example is very much in the spirit of Schachermayer [19].

Example 11. There is a Dunford-Pettis operator from $L_{1}[0,1]$ into $c_{0}$ that is not nearly representable.

Proof. Let $G$ be the Cantor group $\{-1,1\}^{N}$. Let $\left(\alpha_{n}\right)$ be a sequence of positive real numbers such that $\lim _{n} \alpha_{n}=\frac{1}{2}$ but $\sum_{n=1}^{\infty}\left(2 \alpha_{n}-1\right)^{2}=\infty$. Let $\lambda_{n}$ be the measure on $\{-1,1\}$ such that $\lambda_{n}\{+1\}=\alpha_{n}$ and $\lambda_{n}\{-1\}=1-\alpha_{n}$, and let $\lambda=\Pi_{n} \lambda_{n}$. By one of Kakutani's many beautiful theorems (see Diestel-Uhl [5, p. 92]), the measure $\lambda$ and Haar measure $\sigma$ on $G$ are mutually singular since $\sum_{n=1}^{\infty}\left(2 \alpha_{n}-1\right)^{2}=\infty$. Therefore the operator $D_{\lambda}: L_{1}(G, \sigma) \rightarrow L_{1}(G, \sigma)$ defined by $D_{\lambda} f=f * \lambda$ for $f \in L_{1}(G, \sigma)$ is not Bochner representable (DiestelUhl [5, p. 93]). On the other hand, because $\lim _{n} \alpha_{n}=\frac{1}{2}$, the operator $D_{\lambda}$ is Dunford-Pettis (Diestel-Uhl [5, p. 93]).

Following Schachermayer [19], we are going to construct a sequence $\left(g_{n}\right)$ in $L^{\infty}(G, \sigma)$ such that

(i) $0 \leq g_{n} \leq 1$ for all $n=1,2, \ldots$;

(ii) $\lim _{n} g_{n}=0$ in $\sigma$-measure;

(iii) $\varlimsup_{\lim _{n}}\left(D_{\lambda} g_{n}\right)(t)>0$ for each $t \in G$.

Here is the construction of the sequence $\left(g_{n}\right)$ :

Since $\sigma$ and $\lambda$ are mutually singular, there is for each positive integer $n$ a compact set $K_{n} \subseteq G$ such that $\lambda\left(K_{n}\right)>\frac{1}{2}$ and $\sigma\left(K_{n}\right) \leq 1 / n$. Let $f_{n}: G \rightarrow$ $[0,1]$ be a continuous function that equals 1 on $K_{n}$ and 0 on a set of $\sigma$-measure $1-2 / n$. Note that $D_{\lambda} f_{n}$ is continuous on $G$ and compute

$$
D_{\lambda} f_{n}(e)=\int_{G} f_{n} d \lambda>\frac{1}{2}
$$

where $e=(1,1,1,1, \ldots) \in G$. By the continuity of the function $D_{\lambda} f_{n}$, there is a neighborhood $V_{n}$ of $e$ such that

$$
D_{\lambda} f_{n}(t)>\frac{1}{2} \text { for all } t \in V_{n} \text {. }
$$

Because $G$ is compact there are finitely many translations of the function $D_{\lambda} f_{n}$ such that the supremum of the finitely many translations is greater than $\frac{1}{2}$ on all of $G$. Since the convolution operator $D_{\lambda}$ commutes with translations, we see 
that there are finitely many translations $f_{n}^{(1)}, f_{n}^{(2)}, \ldots, f_{n}^{\left(m_{n}\right)}$ of the function $f_{n}$ such that

$$
\sup \left\{D_{\lambda} f_{n}^{(i)}(t): 1 \leq i \leq m_{n}\right\}>\frac{1}{2}
$$

for each $t \in G$. Now define $g_{1}=f_{1}^{(1)}, g_{2}=f_{1}^{(2)}, \ldots, g_{m_{1}}=f_{1}^{\left(m_{1}\right)}, g_{m_{1}+1}=$ $f_{2}^{(1)}, \ldots, g_{m_{1}+m_{2}}=f_{s}^{\left(m_{2}\right)}, \ldots$, and learn that the sequence $\left(g_{n}\right)$ satisfies the desired Properties (i), (ii) and (iii).

Define $T: L_{1}(G, \sigma) \rightarrow c_{0}$ by

$$
T f=\left(\int_{G} f g_{n} d \mu\right)_{n=1}^{\infty}
$$

Since $\lim _{n} g_{n}=0$ in measure, it follows that $T$ is a Dunford-Pettis operator (Diestel-Uhl [5, p. 75]). On the other hand, for each $n$

$$
\left(T \circ D_{\lambda} f\right)_{n}=\int_{G} D_{\lambda} f g_{n} d \mu=\int_{G} f D_{\lambda} g_{n} d \mu
$$

since $D_{\lambda}$ is "selfadjoint". Since $\varlimsup_{n} D_{\lambda} g_{n}>0$ at all points of $G$, we see that $T$ is a Dunford-Pettis operator from $L_{1}(G, \sigma)$ to $L_{1}(G, \sigma)$ that is not nearly representable. Since $L_{1}[0,1]$ is isometric to $L_{1}(G, \sigma)$, the proof is complete.

The interested reader might wonder why we have dwelled on operators from $L_{1}[0,1]$ into $c_{0}$ in the above examples. The answer is easy. The following corollaries and most of the next section show in many cases that unless a friendly copy of $c_{0}$ is available, then $M_{0}$-continuous operators and nearly representable operators are a priori Bochner representable.

Corollary 12. If $X$ is a Banach space containing no copy of $c_{0}$, then any bounded linear operator $T: L_{1}[0,1] \rightarrow X$ that is $M_{0}$-continuous is weakly compact and hence Bochner representable.

Proof. According to the proof of Theorem 9, such an operator $T$ admits a factorization $T=L V$ where $L: C[0,1] \rightarrow X$ is a bounded linear operator and $V: L_{1}[0,1] \rightarrow C[0,1]$ is the Volterra operator. Since $X$ contains no copy of $c_{0}$, an old theorem of Pelczynski's (see Diestel-Uhl [5, VI.2.15]) shows that the operator $L$ is weakly compact, and hence so is $T$. Since weakly compact operators from $L_{1}[0,1]$ into any Banach space are Bochner representable (Diestel-Uhl [5, III.2.12]), the proof is complete.

Actually the conclusion of Corollary 12 can be strengthened if we use an alternate proof. Take a bounded linear operator $T: L_{1}[0,1] \rightarrow X$ that is $M_{0}$ continuous. Write $T=L \circ V$ where the operators $L$ and $V$ are exactly those specified above. Assume $c_{0}$ does not embed in $X$ and use Pelczynski's theorem and the Bartle-Dunford-Schwartz theorem (Diestel-Uhl [5, V.2.5]) to find an $X$-valued countably additive vector measure $G$ on the Borel subsets of $[0,1]$ such that

$$
L \phi=\int_{[0,1]} \phi d G \text { for all } \phi \in C[0,1]
$$


Compute the action of $T=L \circ V$; viz

$$
T f=\int_{0}^{1}\left(\int_{0}^{t} f d \mu\right) d G(t)=\int_{0}^{1} f(s) \int_{s}^{1} d G(t) d \mu(s) .
$$

(The interchange of the order of integration is valid because if one applies a bounded linear functional on $X$ to both integrals, then Fubini's theorem guarantees that the resulting integrals are equal.) Defining $g(s)=\int_{s}^{1} d G(t)$ and note that since $X$ contains no copy of $c_{0}$, the function $g$ has left- and right-hand limits at each point.

To see why this is true, fix $\bar{t} \in[0,1]$ and let $t_{n}$ be an increasing sequence in $(0,1)$ with limit $\bar{t}$. Now

$$
\begin{aligned}
g\left(t_{n}\right) & =g\left(t_{0}\right)+\sum_{i=1}^{n} g\left(t_{i}\right)-g\left(t_{i-1}\right) \\
& =g\left(t_{0}\right)+\sum_{i=1}^{n} \int_{t_{i}}^{1} d G-\int_{t_{i-1}}^{1} d G \\
& =g\left(t_{0}\right)-\sum_{i=1}^{n} G\left[t_{i-1}, t_{i}\right) .
\end{aligned}
$$

Since $G$ is countably additive, the series $\sum_{i=1}^{\infty} G\left[t_{i-1}, t_{i}\right)$ is norm convergent and hence $\lim _{n} g\left(t_{n}\right)$ exists. Just as in the scalar case, it follows that $g$ is regulated; i.e., the function $g$ is the uniform limit of functions of the form $\sum_{i=1}^{n} x_{i} \chi_{I_{i}}$ where $x_{i} \in X$, the $I_{i}$ 's are subintervals of $[0,1]$ and $n$ is a positive integer. In particular, the function $g$ has a relatively norm compact range. This shows that $T f=\int_{0}^{1} f g d \mu$ and since $g$ has a relatively norm compact range that $T$ is a compact operator (Diestel-Uhl [5, III.2.2]).

This discussion proves the following strengthening of Corollary 12 .

Corollary 13. Suppose $X$ contains no copy of $c_{0}$. If $T: L_{1}[0,1] \rightarrow X$ is a bounded linear operator that is $M_{0}$-continuous, then $T: L_{1}[0,1] \rightarrow X$ is a compact operator.

We note that $M_{0}$-continuous operators can also be successfully studied via the theorems found in Weis [20]. For more on this, see Petrakis [17].

\section{3. $L_{1}[0,1]$ HAS THE NRNP}

This section is devoted to proving our main result (Theorem 15) that $L_{1}[0,1]$ has the near Radon-Nikodym property and consequently that any Banach lattice not containing a copy of $c_{0}$ also has the NRNP.

For the purpose of this section, let $\mathbf{T}$ be the unit circle, let $I=[0,1]$, let $\mu$ denote Lebesgue measure on either $\mathbf{T}$ or $I$ and let $M(\mathbf{T})$ and $M[0,1]$ be the Banach space of regular Borel signed measures on $\mathbf{T}$ and $[0,1]$ respectively. Let $\lambda$ be a function on $\mathbf{T}$ with values in the nonnegative singular measures in $M[0,1]$ that satisfies $\lambda(x)([0,1]) \leq 1$ for all $x$ in $\mathbf{T}$, and for which the 
function $x \rightarrow \lambda(x)(E)$ is measurable for each Borel set $E$ in $[0,1]$. For a nonnegative $\sigma$ in $M(T)$, define $T_{\sigma}(\lambda): T \rightarrow M[0,1]$ by

$$
T_{\sigma}(\lambda)(x)(E)=\int_{\mathbf{T}} \lambda(x+t)(E) d \sigma(t)
$$

For a positive increasing function $h:(0, \infty) \rightarrow R$ such that $h\left(0^{+}\right)=0$, define for a subset $A$ of $\mathrm{T}$

$$
M^{h}(A)=\inf \sum_{i=1}^{\infty} h\left(\operatorname{diam} A_{i}\right)
$$

where the infimum is taken over all coverings $\left(A_{i}\right)_{i=1}^{\infty}$ of the set $A \subseteq \mathbf{T}$. If $M^{h}(A)=0$, we say $A$ has Hausdorff $h$-measure 0 .

The following lemma is the basis for the theorems of this section.

Lemma 14. There exists a positive increasing function $h:(0, \infty) \rightarrow R$ such that $h\left(0^{+}\right)=0$ with the property that if $\sigma \in M(\mathbf{T})$ has closed support $S$ with $M^{h}(S)=0$, then $T_{\sigma}(\lambda)(x)$ is singular for almost all $x$ in $\mathbf{T}$.

Proof. Fix $K \geq 1$ and $x \in \mathrm{T}$. For each $N=1,2,3, \ldots$ define a closed set $E(N, K, x) \subseteq I$ as follows. Let $j=\left[2^{N} / K\right]$ (greatest integer function) and consider all unions

$$
\bigcup_{i=1}^{j}\left[\frac{m_{i}}{2^{N}}, \frac{m_{i}+1}{2^{N}}\right] \text { for } 0 \leq m_{i} \leq 2^{N}-1 ;
$$

in other words, take all possible unions of $j$ dyadic intervals of length $2^{-N}$. Choose $E(N, K, x)$ among these unions to maximize the value of $\lambda(x)$. If there is a tie, choose sets to the left. This produces a measurable function $x \rightarrow \lambda(x)(E(N, K, x))$ of $\mathbf{T}$ into $[0,1]$ such that

$$
\lim _{N \rightarrow \infty} \lambda(x)(E(N, K, x))=\lambda(x)(I) \text {. }
$$

To see this convergence, use the singularity of $\lambda(x)$ to get a compact subset $C$ of $I$ such that $\lambda(x)(C)>\lambda(x)(I)-\varepsilon$ but $\mu(C)=0$. Now approximate $C$ by dyadic intervals and invoke the maximality condition in the definition of $E(N, K, x)$.

By Egorov's theorem, there is a set $B_{K}$ with $\mu\left(T \backslash B_{K}\right)<1 / K^{2}$ such that the convergence is uniform on $B_{K}$. Thus there exists $N_{K}$ such that

$$
\lambda(x)\left(E\left(N_{K}, K, x\right)\right) \geq \lambda(x)(I)-1 / K^{2}
$$

for all $x$ in $B_{K}$. There is no trouble in rearranging matters so that $N_{1} \leq N_{2} \leq$ $N_{3} \leq \cdots$.

Again fix $K$. The construction of $E\left(N_{K}, K, x\right)$ chooses unions from a finite collection of sets that are independent of $x$. Thus the function $E\left(N_{K}, K, \cdot\right)$ has only finitely many values. With the help of Lusin's theorem, choose a closed set $C_{K} \subseteq \mathbf{T}$ such that $\mu\left(\mathbf{T} \backslash C_{K}\right)<1 / K^{2}$ and $E\left(N_{K}, K, \cdot\right)$ is continuous on $C_{K}$. Accordingly, the function $E\left(N_{K}, K, \cdot\right)$ is locally constant on $C_{K}$. Thus there 
exists for each $K$ a number $\eta_{K}>0$ such that $E\left(N_{K}, K, x\right)=E\left(N_{K}, K, y\right)$ whenever $|x-y|<\eta_{K}$ and $x, y \in C_{K}$. If necessary, trim the $\eta_{K}$ 's so that $1>\eta_{1}>\eta_{2}>\cdots$ and $\lim _{K} \eta_{K}=0$.

Now we are ready to specify $h$. Choose a positive continuous function $h$ on $(0, \infty)$ such that $h\left(0^{+}\right)=0$ and $h\left(\eta_{K}\right)=1 / K$ for each $K$.

For $j=1,2,3, \ldots$ define

$$
\lambda_{j}(x)= \begin{cases}\lambda(x) & \text { if } x \in \bigcap_{K \geq j} C_{K} \\ 0 & \text { otherwise }\end{cases}
$$

and note, first, that $\lim _{j} \lambda_{j}(x)=\lambda(x)$ in the total variation norm, and, second, that $\lambda_{j}(x)$ increases to $\lambda(x)$ for all $x$. From the monotone convergence of the $\lambda_{j}$ 's to $\lambda$, it follows that

$$
\lim _{j} T_{\sigma}\left(\lambda_{j}\right)(x)=T_{\sigma}(\lambda)(x)
$$

in the total variation norm for almost all $x$.

Now if we can show that for each $j$ the measures $T_{\sigma}\left(\lambda_{j}\right)(x)$ are singular for almost all $x$, then we will have proved the lemma because the set of singular measures is closed in the total variation norm.

To this end, fix $j$. For each $p \in \mathbf{N}$ choose $\varepsilon_{p} \leq \min \left\{1 / p^{2}, h\left(\eta_{j+p}\right)\right\}$. Recall that $S$, the closed support of $\sigma$, has Hausdorff $h$-measure 0 . Thus we can write $S=\bigcup_{q=1}^{\infty} S_{q}^{p}$ with $\sum_{q} h\left(\operatorname{diam} S_{q}^{p}\right)<\varepsilon_{p}$. We can first choose the sets $S_{q}^{p}$ to be closed, and then to be measurable with $S_{q_{1}}^{p} \cap S_{q_{2}}^{p}=\phi$ for $q_{1} \neq q_{2}$. For ease of notation, we will write $S_{q}$ for $S_{q}^{p}$.

Note that $\operatorname{diam}\left(S_{q}\right) \geq \eta_{j+p}$ forces $h\left(\operatorname{diam} S_{q}\right) \geq h\left(\eta_{j+p}\right) \geq \varepsilon_{p}$, an impossibility. Hence $\operatorname{diam}\left(S_{q}\right)<\eta_{j+p}$ for each $S_{p}^{q}$. For each $q$ choose $K(q)$ $(=K(q, p)) \geq j+p$ such that

and define

$$
\eta_{K(q)+1}<\operatorname{diam} S_{q} \leq \eta_{K(q)} \leq \eta_{j+p}
$$

$$
\begin{gathered}
\lambda_{j, q}^{(1)}(x)(E)=\lambda_{j}(x)\left(E \cap E\left(N_{K(q)}, K(q), x\right) \geq 0,\right. \\
\lambda_{j, q}^{(2)}(x)=\lambda_{j}(x)-\lambda_{j, q}^{(1)}(x) \geq 0 .
\end{gathered}
$$

Let

$$
\nu_{q}(x)(E)=\int_{S_{q}} \lambda_{j, q}^{(2)}(x+t)(E) d \sigma(t)
$$

and compute

$$
\begin{aligned}
\int_{\mathbf{T}} \nu_{q}(x)(I) d x & =\int_{\mathbf{T}} \int_{S_{q}} \lambda_{j, q}^{(2)}(x+t)(I) d \sigma(t) d x \\
& =\int_{S_{q}} \int_{\mathbf{T}} \lambda_{j, q}^{(2)}(x+t)(I) d x d \sigma(t) \\
& =\int_{S_{q}} \int_{\mathbf{T}} \lambda_{j, q}^{(2)}(x)(I) d x d \sigma(t) \\
& =\sigma\left(S_{q}\right) \int_{\mathbf{T}} \lambda_{j, q}^{(2)}(x)(I) d x .
\end{aligned}
$$


Now

$$
\begin{aligned}
\int_{\mathbf{T}} \lambda_{j, q}^{(2)}(x)(I) d x & =\int_{B_{K(q)}} \lambda_{j, q}^{(2)}(x)(I) d x+\int_{\mathbf{T} \backslash B_{K(q)}} \lambda_{j, q}^{(2)}(x)(I) d x \\
& \leq \frac{1}{K(q)^{2}} \mu(\mathbf{T})+1 \cdot \mu\left(\mathbf{T} \backslash B_{K(q)}\right) \leq \frac{2 \pi+1}{K(q)^{2}},
\end{aligned}
$$

by the definition of the $B_{K}$ 's and $N_{K}$ 's. This proves

$$
\int_{\mathbf{T}} \nu_{q}(x)(I) d x \leq \frac{2 \pi+1}{K(q)^{2}} \sigma\left(S_{q}\right) .
$$
that

If we let $\tau_{p}(x)=\sum_{q=1}^{\infty} \nu_{q}(x)=\sum_{q=1}^{\infty} \int_{S_{q}} \lambda_{j, q}^{(2)}(x+t)(E) d \sigma(t)$ then we see

$$
\begin{aligned}
\int_{\mathbf{T}} \tau_{p}(x)(I) d x & \leq(2 \pi+1) \sum_{q=1}^{\infty} \frac{1}{K(q)^{2}} \sigma\left(S_{q}\right) \\
& \leq(2 \pi+1) \sum_{q=1}^{\infty} \frac{1}{(j+p)^{2}} \sigma\left(S_{q}\right) \leq \frac{2 \pi+1}{(j+p)^{2}} \sigma(\mathbf{T}) .
\end{aligned}
$$

Next consider the measure

$$
\beta_{q}(x)(E)=\int_{S_{q}} \lambda_{j, q}^{(1)}(x+t)(E) d \sigma(t) .
$$

Fix $x \in \mathbf{T}$ and consider the set

$$
P_{q}=\left\{t \in S_{q}: \lambda_{j, q}^{(1)}(x+t)(I)>0\right\} .
$$

Note that if $t \in P_{q}$ then $x+t \in \bigcap_{K \geq j} C_{K}$, for if not, then $\lambda_{j}(x+t)=0$ and hence

$$
0=\lambda_{j}(x+t)\left(E\left(N_{K(q)}, K(q), x\right)\right)=\lambda_{j, q}^{(1)}(x+t)(I) .
$$

Since $K(q) \geq j+p \geq j$, we see that $t \in P_{q}$ forces $x+t \in C_{K(q)}$. Similarly, if $t^{\prime} \in S_{q}$ and $\lambda_{j, q}^{(1)}\left(x+t^{\prime}\right)(I)>0$, then $x+t^{\prime} \in C_{K(q)}$.

Now

Let $t$ and $t^{\prime}$ be in $S_{q}$. Because $\operatorname{diam}\left(S_{q}\right)<\eta_{K(q)}$, we have $\left|t-t^{\prime}\right|<\eta_{K(q)}$.

$$
\lambda_{j, q}^{(1)}(x+t)(I)=\lambda_{j}(x+t)\left(E\left(N_{K(q)}, K(q), x+t\right)\right)
$$

and

$$
\lambda_{j, q}^{(1)}\left(x+t^{\prime}\right)(I)=\lambda_{j}\left(x+t^{\prime}\right)\left(E\left(N_{K(q)}, K(q), x+t^{\prime}\right)\right) \text {. }
$$

If these two values are both strictly positive, then $x+t$ and $x+t^{\prime}$ both belong to $C_{K(q)}$ and

$$
\left|(x+t)-\left(x+t^{\prime}\right)\right|=\left|t-t^{\prime}\right|<\eta_{K(q)} .
$$

Hence by local constancy

$$
E\left(N_{K(q)}, K(q), x+t\right)=E\left(N_{K(q)}, K(q), x+t^{\prime}\right) .
$$


Now $\lambda_{j, q}^{(1)}(x+t)$ is concentrated on $E\left(N_{K(q)}, K(q), x+t\right)$. But these sets are the same for all $t \in P_{q}$. Thus the measure

$$
\beta_{q}(x)(E)=\int_{S_{q}} \lambda_{j, q}^{(1)}(x+t)(E) d \sigma(t)=\int_{P_{q}} \lambda_{j, q}^{(1)}(x+t)(E) d \sigma(t)
$$

is concentrated on some set $E\left(N_{K(q)}, K(q), x+\bar{t}\right)$ for some fixed $\bar{t} \in S_{q}$. Note that

$$
\mu\left(E\left(N_{K(q)}, K(q), x+\bar{t}\right)\right) \leq \frac{2^{N_{K(q)}}}{K(q)} \cdot \frac{1}{2^{N_{K(q)}}}
$$

because there are at most $2^{N_{K(q)}} K(q)^{-1}$ intervals each of size $2^{-N_{K(q)}}$ that make up this set.

Recall $\operatorname{diam}\left(S_{q}\right) \geq \eta_{K(q)+1}$ whence

$$
h\left(\operatorname{diam}\left(S_{q}\right)\right) \geq h\left(\eta_{K(q)+1}\right)=\frac{1}{K(q)+1} .
$$

Therefore the measure

$$
\gamma_{p}(x)(E)=\sum_{q=1}^{\infty} \int_{S_{q}} \lambda_{j, q}^{(1)}(x+t)(E) d \sigma(t)
$$

is for each $x$ concentrated on a set of Lebesgue measure no greater than

$$
\sum_{q=1}^{\infty} \frac{1}{K(q)} \leq \sum_{q=1}^{\infty} \frac{2}{K(q)+1} \leq 2 \sum_{q=1}^{\infty} h\left(\operatorname{diam} S_{q}\right)<2 \varepsilon_{p} .
$$

We are almost done. Let us consolidate our position. We have a function $T_{j}$ $\left(=T_{\sigma}\left(\lambda_{j}\right)\right)$ mapping $\mathbf{T}$ into $M[0,1]$ and a sequence $\left(\varepsilon_{p}\right)$ with $\sum_{p=1}^{\infty} \varepsilon_{p}<\infty$ such that for each $p$

$$
0 \leq T_{j}(x)=\gamma_{p}(x)+\tau_{p}(x)
$$

where $\gamma_{p}(x)$ and $\tau_{p}(x)$ are nonnegative measures such that

(1) $\gamma_{p}(x)$ is concentrated on some set $E_{p}(x)$;

(2) the Lebesgue measure $\mu\left(E_{p}(x)\right)<2 \varepsilon_{p}$; and

(3) $\int_{\mathrm{T}} \tau_{p}(x)(I) d x \leq A /(j+p)^{2}$.

We want to show that $T_{j}(x)$ is singular for almost all $x$. From (3), we see $\lim _{p} \tau_{p}(\cdot)(I)=0$ in $L_{1}(\mathbf{T})$. Passing to a subsequence if necessary, we may assume $\lim _{p} \tau_{p}(x)(I)=0$ for all $x \in \mathbf{T} \backslash C$ where $C$ is a Lebesgue null set. Now for each $k \in \mathbf{N}$ observe that for any measurable set $E$ such that $E \cap$ $\left(\bigcup_{p \geq k} E_{p}\right)=\phi$ we have

$$
0 \leq T_{j}(x)(E)=\gamma_{p}(x)(E)+\tau_{p}(x)(E)=0+\tau_{p}(x)(E)
$$

for all $p \geq k$. Hence

$$
0 \leq T_{j}(x)(E) \leq \lim _{p} \tau_{p}(x)(E) \leq \lim _{p} \tau_{p}(x)(I)=0
$$


for each $x \in \mathbf{T} \backslash C$. This proves that for each $x \in \mathbf{T} \backslash C$ the measure $T_{j}(x)$ is concentrated on the Lebesgue null set $\bigcap_{k=1}^{\infty} \bigcup_{p \geq k} E_{p}(x)$, and this finally completes the proof of the lemma.

Lemma 14 sets up the main theorem of this paper. For operators mapping $L_{1}[0,1]$ into $L_{1}[0,1]$, this theorem represents a strengthening of a theorem of Bourgain [1] who proved a similar theorem for operators that are not DunfordPettis.

Theorem 15. If $T: L_{1}[0,1] \rightarrow L_{1}[0,1]$ is not Bochner representable, then there is a Dunford-Pettis operator $D: L_{1}[0,1] \rightarrow L_{1}[0,1]$ such that $T \circ D$ is not Bochner representable. Consequently, any nearly representable operator from $L_{1}[0,1]$ into $L_{1}[0,1]$ is Bochner representable and the space $L_{1}[0,1]$ has the near Radon-Nikodym property.

The proof of this theorem requires the following additional lemmas. Probably these lemmas are well known to some, but we could find no specific reference.

Lemma 16. The map $\sigma \rightarrow \sigma^{+}$is weak ${ }^{*}$-norm Borelian on $M[0,1]$.

Proof. Let $P$ be the set of nonnegative functions in the closed unit ball of $C[0,1]$ and pick a sequence $\left(f_{n}\right)_{n=1}^{\infty}$ in $P$ that is dense in $P$. Let $f_{0} \equiv 0$.

Fix $\sigma \in M[0,1]$ and define

$$
a_{n}(\sigma)=\int_{[0,1]} f_{n} d \sigma \text { and } A_{n}(\sigma)=\max \left(a_{0}(\sigma), \ldots, a_{n}(\sigma)\right) .
$$

Observe that $0 \leq A_{n}(\sigma) \leq \sigma^{+}([0,1])$ and that $\lim _{n} A_{n}(\sigma)=\sigma^{+}([0,1])$. Choose nonnegative integers $m_{1}, \ldots, m_{n}$ such that $0 \leq m_{n} \leq n$ and

$$
a_{m_{n}}(\sigma)=A_{n}(\sigma) \text {. }
$$

(Choose $m_{n}$ to be the least possible if there is a tie.) Observe that $m_{1}, \ldots, m_{n}$ are measurable functions of $\sigma$ and define signed measures $\tau_{n}$ by

$$
\tau_{n}=f_{m_{n}} \cdot \sigma
$$

Note that

$$
\sigma^{+}([0,1])=\lim _{n} A_{n}(\sigma)=\lim _{n} \tau_{n}([0,1])=\lim _{n} \int_{[0,1]} f_{m_{n}} d \sigma .
$$

Because $0 \leq f_{m_{n}} \leq 1$, we infer that

$$
\lim _{n} f_{m_{n}}=1 \text { a.e. }\left[\sigma^{+}\right]
$$

and

$$
\lim _{n} f_{m_{n}}=0 \text { a.e. }\left[\sigma^{-}\right] .
$$

Hence $\lim _{n} \tau_{n}=\sigma^{+}$in the variation norm of $M[0,1]$. Since each $\tau_{n}$ is a Borel measurable function of $\sigma$, we see that $\sigma^{+}$is a Borel measurable function of $\sigma$. This completes the proof of the lemma.

Actually, this lemma can be improved with just a little more work. 
Theorem 17. The mapping $\sigma \rightarrow \sigma^{+}$is weak ${ }^{*}$-norm Baire class 1 on $M[0,1]$. Proof. We have to show the process of taking $\sigma$ to $\sigma^{+}$is a norm limit of continuous maps of $M[0,1]$ into itself.

To this end, take $\sigma \in M[0,1]$, maintain the same notation as in the proof of the last lemma, and define

$$
h(u)= \begin{cases}1-|u| & \text { for }-1 \leq u \leq 1 \\ 0 & \text { for }|u|>1\end{cases}
$$

Fix a positive integer $n$ and set

$$
\alpha_{j}(\sigma)=h\left(n a_{j}(\sigma)-n A_{n}(\sigma)\right)
$$

for each $j=1,2, \ldots, n$. Note that each $\alpha_{j}(\sigma)$ is a continuous function of $\sigma$. Let

$$
g_{n}=\frac{\sum_{j=1}^{n} \alpha_{j}(\sigma) f_{j}}{\sum_{j=1}^{n} \alpha_{j}(\sigma)}=\frac{\sum_{a_{j}(\sigma) \geq A_{n}(\sigma)-1 / n} \alpha_{j}(\sigma) f_{j}}{\sum_{a_{j}(\sigma) \geq A_{n}(\sigma)-1 / n} \alpha_{j}(\sigma)}
$$

and note that

(i) $g_{n}$ depends continuously on $\sigma$;

(ii) $0 \leq g_{n} \leq 1$; and

(iii) $\int_{[0,1]} g_{n} d \sigma \geq A_{n}(\sigma)-1 / n$.

Define $\gamma_{n} \in M[0,1]$ by $\gamma_{n}=g_{n} \cdot \sigma$ and note that just as in the proof of the last lemma, $\lim _{n} \gamma_{n}=\sigma^{+}$in the variation norm of $M[0,1]$. Since each $\gamma_{n}$ depends continuously on $\sigma$, the proof is complete.

The Lebesgue decomposition of $\sigma \in M[0,1]$ finds unique measures $\sigma_{\text {ac }}$ and $\sigma_{\mathrm{s}}$ in $M[0,1]$ such that $\sigma_{\mathrm{ac}}$ is absolutely continuous and $\sigma_{\mathrm{s}}$ is singular (with respect to Lebesgue measure) with the property that $\sigma=\sigma_{\mathrm{ac}}+\sigma_{\mathrm{s}}$.

Corollary 18. The mapping $\sigma \rightarrow \sigma_{\mathrm{s}}$ is of weak ${ }^{*}$-norm Baire class 2 on $M[0,1]$. Proof. Let $\mu$ be Lebesgue measure and note that for a positive $\sigma$ in $M[0,1]$, we have

$$
\sigma_{\mathrm{s}}=\lim _{n}(\sigma-n \mu)^{+}
$$

We are now ready for the proof of Theorem 15.

Proof of Theorem 15. Let $\sigma$ be in $M[0,1]$ and define the convolution operator

$$
D_{\sigma} f(x)=\int_{\mathbf{T}} f(x-y) d \sigma(y) \text {. }
$$

The operator $D_{\sigma}: L_{1}(\mathrm{~T}) \rightarrow L_{1}(\mathrm{~T})$ is Dunford-Pettis if and only if the Fourier coefficients of $\sigma$ vanish at infinity; that is, $\lim _{k} \hat{\sigma}(k)=0$ (see Diestel-Uhl [5, p. 93]). Our object is to take a non-Bochner representable operator $S: L_{1}(\mathbf{T}) \rightarrow$ $L_{1}[0,1]$ and find $\sigma$ in $M[0,1]$ such that $D_{\sigma}$ is Dunford-Pettis but $S \circ D_{\sigma}$ is not representable. 
According to Dunford-Schwartz [8, VI.8.9] there is a correspondence between operators $S: L_{1}(\mathbf{T}) \rightarrow L_{1}[0,1]$ and functions $\lambda: \mathbf{T} \rightarrow M[0,1]$ such that

(1) for each Borel measurable $E \subseteq[0,1]$, the map $x \rightarrow \lambda(x)(E)$ is measurable;

(2) $0 \leq|\lambda(x)| \leq\|S\| \leq 1$ for all $x$;

(3) for each measurable $A \subseteq \mathbf{T}$, the measure $E \rightarrow \int_{A} \lambda(x)(E) d x$ is regular and $\mu$-continuous; and

(4) $S f=d / d \mu \int_{\mathrm{T}} \lambda(x)(\cdot) f(x) d x$ for all $f \in L_{1}(\mathbf{T})$.

We shall call $\lambda$ the kernel of $S$. Note that $\lambda$ is essentially uniquely defined.

Now fix $S: L_{1}(T) \rightarrow L_{1}[0,1]$ with kernel $\lambda$, fix $\sigma$ in $M[0,1]$, and compute

$$
\begin{aligned}
S \circ D \sigma f & =\frac{d}{d \mu} \int_{\mathbf{T}} \lambda(x)(\cdot) D_{\sigma} f(x) d x=\frac{d}{d \mu} \int_{\mathbf{T}} \lambda(x)(\cdot) \int_{\mathbf{T}} f(x-y) d \sigma(y) d x \\
& =\frac{d}{d \mu} \int_{\mathbf{T}} \int_{\mathbf{T}} \lambda(x)(\cdot) f(x-y) d \sigma(y) d x \\
& =\frac{d}{d \mu} \int_{\mathbf{T}} \int_{\mathbf{T}} \lambda(x+y)(\cdot) f(x) d \sigma(y) d x \\
& =\frac{d}{d \mu} \int_{\mathbf{T}} f(x)\left[\int_{\mathbf{T}} \lambda(x+y)(\cdot) d \sigma(y)\right] d x .
\end{aligned}
$$

Therefore the kernel of $S \circ D_{\sigma}$ is $\int_{\mathrm{T}} \lambda(x+y) d \sigma(y)$.

Now write the Lebesgue decompositions

$$
\lambda(x)(\cdot)=\lambda_{\mathrm{ac}}(x)(\cdot)+\lambda_{\mathrm{s}}(x)(\cdot) .
$$

It is not hard to see that $S$ is Bochner representable if and only if $\lambda_{s}(x)=0$ for almost all $x$ in $\mathbf{T}$.

The measurability guaranteed by Corollary 18 guarantees that $\lambda_{\mathrm{ac}}(x)(\cdot)$ and $\lambda_{\mathrm{s}}(x)(\cdot)$ are kernels of operators $S_{\mathrm{ac}}: L_{1}(\mathrm{~T}) \rightarrow L_{1}[0,1]$ and $S_{\mathrm{s}}: L_{1}(\mathrm{~T}) \rightarrow$ $L_{1}[0,1]$ such that $S=S_{\mathrm{ac}}+S_{\mathrm{s}}$. Moreover, if $\sigma$ is any measure in $M(\mathrm{~T})$, then $S_{\mathrm{ac}} \circ D_{\sigma}$ is Bochner representable because $S_{\mathrm{ac}}$ is Bochner representable. Thus to prove the theorem, it suffices to take $S: L_{1}(T) \rightarrow L_{1}[0,1]$ such that $S_{\mathrm{s}} \neq 0$ and find a $\sigma_{0}$ in $M(\mathrm{~T})$ such that the kernel of $S_{\mathrm{s}} \circ D_{\sigma}$ takes singular values almost everywhere.

To this end, suppose $S_{\mathrm{s}} \neq 0$ and has kernel $\lambda_{\mathrm{s}}$. Note that the variation $\left|\lambda_{s}\right|(x)$ is singular for almost all $x$ in $T$. According to Lemma 14, there is a positive increasing function $h$ on $(0, \infty)$ such that $h\left(0^{+}\right)=0$ with the property that if $\sigma$ is a nonnegative member of $M(T)$ that has closed support with Hausdorff $h$-measure 0 , then the measure given by

$$
\int_{\mathbf{T}}\left|\lambda_{\mathrm{s}}\right|(x+t)(\cdot) d \sigma(t)
$$

is singular for almost all $x$. By a theorem of Isasev-Musatov (for an exposition and generalization of this theorem, see Kaufman [10]), there is a closed set $B \subseteq$ T with $M^{h}(B)=0$ and a nonnegative measure $\sigma$ supported on $B$ such that the Fourier coefficients vanish at infinity; that is, $\lim _{k} \hat{\sigma}(k)=0$. Consequently, 
for this measure $\sigma$, the measure $\int_{\mathrm{T}}\left|\lambda_{\mathrm{s}}\right|(x+t) d \sigma(t)$ is singular for almost all $x$. Therefore, of course, the measure $\int_{\mathrm{T}} \lambda_{\mathrm{s}}(x+t) d \sigma(t)$ is also singular for almost all $x$.

The only difficulty is that $\int_{\mathrm{T}} \lambda_{\mathrm{s}}(x+t) d \sigma(t)$ might be zero for almost all $x$. To get around this, choose some $k$ such that

$$
\frac{1}{2 \pi} \int_{\mathbf{T}} \lambda_{\mathbf{s}}(x) e^{-i k x} d x \neq 0
$$

(this can be done because $\lambda_{\mathrm{s}}$ is not almost everywhere 0 ). Choose a measurable $u$ with $0<u<1$ such that

$$
\int_{\mathbf{T}} u(y) e^{i k y} d \sigma(y) \neq 0
$$

Now consider the kernel

$$
\bar{\lambda}(x)=\int_{\mathrm{T}} \lambda_{\mathrm{s}}(x+y) u(y) d \sigma(y) .
$$

Again by Lemma 14, this kernel is singular for almost all $x$ in $\mathbf{T}$. In addition,

$$
\begin{aligned}
\int_{\mathbf{T}} \bar{\lambda}(x) e^{-i k x} d x & =\int_{\mathbf{T}} \int_{\mathbf{T}} \lambda_{\mathbf{s}}(x+y) u(y) e^{-i k x} d x d \sigma(y) \\
& =\int_{\mathbf{T}} \int_{\mathbf{T}} \lambda_{\mathbf{s}}(x) u(y) e^{-i k(x-y)} d x d \sigma(y) \\
& =\int_{\mathbf{T}} \lambda_{\mathbf{s}}(x) e^{-i k x} d x \int_{\mathbf{T}} u(y) e^{i k y} d \sigma(y) \neq 0 .
\end{aligned}
$$

Finally, set $\sigma_{0}=u \cdot \sigma$. We still have $\lim _{k} \hat{\sigma}_{0}(k)=0$ and we still have that almost all the values of $\sigma_{0}$ are singular. Hence $D_{\sigma_{0}}$ is a Dunford-Pettis operator and the operator $S_{s} \circ D_{\sigma_{0}}$ has a kernel $\bar{\lambda}$ not a.e. zero but taking almost all its values in the singular measures. This proves that $S_{\mathrm{s}} \circ D_{\sigma_{0}}$ is not Bochner representable and this completes the proof.

Thanks to the theory of semi-embeddings, Theorem 15 can be parlayed into a general theorem for Banach lattices.

Corollary 19. If $X$ is a Banach lattice containing no copy of $c_{0}$, then $X$ has the near Radon-Nikodym property.

Proof. Since operators from $L_{1}[0,1]$ into $X$ have separable ranges, we may assume without loss of generality that $X$ is a separable Banach lattice containing no copy of $c_{0}$. Choose a separable probability space $(\Omega, \Theta, P)$ such that $X$ can be represented as a space of measurable functions such that $L^{\infty}(P) \subseteq X \subseteq$ $L_{1}(P)$ and the canonical (identity) injections are continuous (for an exposition see Lindenstrauss-Tzafriri [12]). According to a theorem of Lotz, Peck and Porta $[13,14]$, the identity injection $J_{X}: X \rightarrow L_{1}(P)$ is a semi-embedding; i.e. the ball $B_{X}$ is closed in $L_{1}(P)$. By a theorem of Bourgain and Rosenthal, an operator $T: L_{1}[0,1] \rightarrow X$ is Bochner representable if and only if the operator $J_{X} \circ T: L_{1}[0,1] \rightarrow L_{1}(P)$ is Bochner representable. Thus $T: L_{1}[0,1] \rightarrow X$ is nearly representable if and only if $J_{X} \circ T$ is nearly representable. Since $L_{1}(P)$ 
is isometric to a subspace of $L_{1}[0,1]$, Theorem 15 applies and reveals that every nearly representable $T: L_{1}[0,1] \rightarrow X$ is Bochner representable. This completes the proof.

Corollary 20. If $X$ is a Banach lattice such that every bounded linear operator $T: L_{1}[0,1] \rightarrow X$ is nearly representable, then $X$ has the Radon-Nikodym property.

Proof. If $c_{0}$ embeds in $X$, then by Example 11, there is a bounded linear operator $T: L_{1}[0,1] \rightarrow X$ that is not nearly representable. Therefore $X$ contains no copy of $c_{0}$. Now appeal to Corollary 19 to see that every bounded linear operator $T: L_{1}[0,1] \rightarrow X$ is representable.

Proved in a similar way is

Corollary 21. If $X$ is a Banach lattice such that every Dunford-Pettis operator $T: L_{1}[0,1] \rightarrow X$ is nearly representable, then $X$ has the Radon-Nikodym property.

Proof. Again refer to Example 11 to see that $c_{0}$ cannot embed in $X$. According to a theorem of Bourgain [1], if every Dunford-Pettis operator $T: L_{1}[0,1] \rightarrow X$ is Bochner representable, then $X$ has the RNP. Thus we can proceed as in the proof of Corollary 20 to prove that $X$ has the Radon-Nikodym property.

The following corollary is an old chestnut. It was originally proved by Peck, Lotz and Porta [13,14] and reproved by Bourgain and Rosenthal [3]. We give yet another proof.

Corollary 22. There is no semi-embedding of $c_{0}$ into any Banach lattice that contains no copy of $c_{0}$. In particular, there is no semi-embedding of $c_{0}$ into $L_{1}[0,1]$.

Proof. Review the proof of Corollary 19 to see that if $X$ is a Banach space that semi-embeds in another Banach space $Y$, then $X$ has the near RadonNikodym property if $Y$ has the near Radon-Nikodym property. This fact proves the corollary because $c_{0}$ fails the near Radon-Nikodym property, but Banach lattices containing no copy of $c_{0}$ have the near Radon-Nikodym property.

Finally we shall look at the near Radon-Nikodym property for the quotient space $L_{1}(\mathrm{~T}) / H_{0}^{1}$. The space $H_{0}^{1}$ is the subspace of the Hardy space $H^{1}$ consisting of functions that vanish at the origin. The same factoring and lifting trick used in the proof of Theorem 7 will also be employed here.

Lemma 23. Let $R: L_{1}(\mathbf{T}) \rightarrow L_{1}(\mathbf{T})$ be a bounded linear operator and let $q: L_{1}(\mathbf{T})$ $\rightarrow L_{1}(\mathrm{~T}) / H_{0}^{1}$ be the quotient map. The composition $q \circ R: L_{1}(\mathrm{~T}) \rightarrow L_{1}(\mathrm{~T}) / H_{0}^{1}$ is Bochner representable if and only if $R: L_{1}(\mathbf{T}) \rightarrow L_{1}(\mathbf{T})$ is Bochner representable.

Proof. If $R$ is Bochner representable, then $q \circ R$ is automatically Bochner representable. 
For the converse, suppose $q \circ R$ is Bochner representable and use the LewisStegall theorem to obtain bounded linear operators $B: L_{1}(\mathbf{T}) \rightarrow l_{1}$ and $A: l_{1} \rightarrow$ $L_{1}(\mathrm{~T}) / H_{0}^{1}$ such that $q \circ R=A \circ B$.

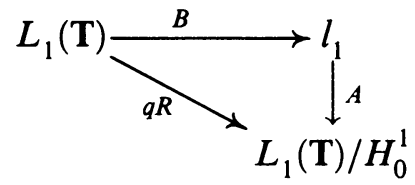

Because $q$ is onto, the lifting property for $l_{1}$ (see Lindenstrauss-Tzafriri [12, p. 107]) guarantees the existence of a bounded linear operator $\tilde{A}: l_{1} \rightarrow L_{1}(\mathrm{~T})$ such that $q \tilde{A}=A$. Hence $q \tilde{A} B=A B=q R$ and therefore $q(R-\tilde{A B})=0$. This means that the range of $R-\widetilde{A B}$ is contained in the separable dual space $H_{0}^{1}$. Since separable duals have the Radon-Nłkodym property (Diestel-Uhl [5, III.3.1]), the operator $R-\widetilde{A B}$ is Bochner representable. Since $\tilde{A} B$ evidently factors through $l_{1}$, it too is Bochner representable, and thus $R=(R-\tilde{A} B)+\widetilde{A} B$ is Bochner representable.

Corollary 24. The space $L_{1}(\mathrm{~T}) / H_{0}^{1}$ has the near Radon-Nikodym property.

Proof. Let $T: L_{1}(\mathrm{~T}) \rightarrow L_{1}(\mathrm{~T}) / H_{0}^{1}$ be nearly representable. We have to show that $T$ is Bochner representable. According to a theorem of Ghoussoub and Rosenthal [9], any bounded linear operator from $L_{1}(\mathrm{~T})$ into $L_{1}(\mathrm{~T}) / H_{0}^{1}$ factors through $L_{1}(\mathbf{T})$ via the quotient map $q: L_{1}(\mathbf{T}) \rightarrow L_{1}(\mathbf{T}) / H_{0}^{1}$. Accordingly, there is a bounded linear operator $R: L_{1}(\mathbf{T}) \rightarrow L_{1}(\mathbf{T})$ such that $T=q \circ R$. Now let $D: L_{1}(\mathbf{T}) \rightarrow L_{1}(\mathbf{T})$ be a Dunford-Pettis operator.

$$
L_{1}(\mathbf{T}) \stackrel{D}{\rightarrow} L_{1}(\mathbf{T}) \stackrel{T}{\rightarrow} L_{L_{1}(\mathbf{T})}^{L_{1}(\mathbf{T}) / H_{0}^{1}}
$$

Since $T$ is nearly representable, the composition $T \circ D$ is Bochner representable and hence $q \circ R \circ D=T \circ D$ is Bochner representable. By Lemma 23, the operator $R \circ D$ is Bochner representable. This shows that $R: L_{1}(\mathbf{T}) \rightarrow L_{1}(\mathbf{T})$ is nearly representable. Since by Theorem 15 , the space $L_{1}(T)$ has the near Radon-Nikodym property, we see that $R$ is itself Bochner representable. Therefore $T=q \circ R$ is also Bochner representable.

A complex Banach space $X$ is said to have the analytic Radon-Nikodym property (ARNP) if every $X$-valued bounded analytic function defined on the open unit disk has radial limits at almost all boundary points. This notion was introduced by Bukhvalov and Danilevich [4] who showed that $L_{1}(T)$ has the analytic Radon-Nikodym property, but that $L_{1}(\mathrm{~T}) / H_{0}^{1}$ fails this property. We see, therefore, that the ARNP and NRNP are different properties. For additional information on the ARNP, see Dowling [6].

Acknowledgement. The authors wish to thank Paulette Saab and Elias Saab for some helpful comments. 


\section{REFERENCES}

1. J. Bourgain, Dunford-Pettis operators on $L_{1}$ and the Radon-Nikodym property, Israel J. Math. 37 (1980), 34-47.

2. _ A characterization of non-Dunford-Pettis operators on $L_{1}$, Israel J. Math. 37 (1980), 48-53.

3. J. Bourgain and H. P. Rosenthal, Applications of the theory of semi-embeddings to Banach space theory, J. Funct. Anal. 52 (1983), 149-188.

4. A. V. Bukhvalov and A. A. Danilevich, Boundary properties of analytic and harmonic functions with values in a Banach space, Mat. Zametki 31 (1982), 203-214 (English translation: Math. Notes 31 (1982), 104-110).

5. J. Diestel and J. J. Uhl, Jr., Vector measures, Math. Surveys, no. 15, Amer. Math. Soc., Providence, R.I., 1977.

6. P. Dowling, The analytic Radon-Nikodym property in Banach spaces, Thesis, Kent State University.

7. _ Representable operators and the analytic Radon-Nikodym property in Banach spaces, Proc. Royal Irish Acad. 85A (1985), 143-150.

8. N. Dunford and J. T. Schwartz, Linear operators, Part I, Interscience, New York, 1957.

9. N. Ghoussoub and H. P. Rosenthal, Martingales, $G_{\delta}$-embeddings and quotients of $L_{1}$, Math. Ann. 264 (1983), 321-332.

10. R. Kaufman, Kronecker sets and metric properties of $M_{0}$-sets, Proc. Amer. Math. Soc., 36 (1972), 519-523.

11. D. R. Lewis, A vector measure with no derivative, Proc. Amer. Math. Soc. 32 (1972), 195-198.

12. J. Lindenstrauss and L. Tzafriri, Classical Banach spaces. I, Springer-Verlag, New York, 1977.

13. H. P. Lotz, N. T. Peck and H. Porta, Semi-embeddings of Banach spaces, Proc. Edinburgh Math. Soc. 22 (1979), 233-240.

14. __ Semi-embeddings in linear topological spaces, Conference on harmonic analysis in honor of Antoni Zygmund (Chicago, Ill., 1981), Wadsworth Math. Series, Belmont, California, 1983, pp. 749-763.

15. M. Petrakis, Nearly representable operators, Ph.D. Thesis, University of Illinois at UrbanaChampaign, 1986.

16. __ Nearly representable operators-general theory, (in preparation).

17. _ Diffuse measures and near representability, (in preparation).

18. L. H. Riddle and J. J. Uhl, Jr., Martingales and the fine line between Asplund spaces and spaces not containing a copy of $l_{1}$, Martingale Theory in Harmonic Analysis and Banach Spaces, Lecture Notes in Math., vol. 339, Springer-Verlag, Berlin, 1981, pp. 145-156.

19. W. Schachermayer, Some remarks on integral operators and equimeasurable sets, (preprint).

20. L. Weis, On the representation of order continuous operators by random measures, Trans. Amer. Math. Soc. 285 (1984), 535-563.

Department of Mathematics, University of Illinois, 1409 W. Green ST., Urbana, IlliNols 61801 (Current address of R. Kaufman, M. Petrakis and J. J. Uhl)

Department of Mathematics and Computer Science, Emory University, Atlanta, GeORGIA 30322 (Current address of L. H. Riddle) 